\title{
METASTASIS CEREBRAL DE ADENOCARCINOMA DE CERVIX
}

\author{
CEREBRAL METASTASIS FROM ADENOCARCINOMA OF CERVIX \\ Álvaro Alzamora-Jiménez', Alejandro Castillo-Gutierrez², Juan Coasaca-Torres²
}

\begin{abstract}
RESUMEN
El cáncer de cuello uterino es el tipo de cáncer más frecuente en la mujer peruana y en la población general. Las metástasis cerebrales de cuello uterino son extremadamente raras con una incidencia de 0.4 a $2.3 \%$ y sólo aproximadamente 100 reportes de casos en la literatura médica. Reportamos un caso de una mujer de 52 años de edad con antecedente de cáncer de cuello uterino que luego de 14 meses de dicho diagnóstico presenta cefalea, vómitos y trastorno de conciencia. En el estudio de RMN cerebral se evidencia una lesión captadora de contraste en la región parieto-occipital derecha. Se realiza craneotomía parietal mínima con asistencia estereotáctica y resección completa del tumor. Según el informe del servicio de Patología se indica metástasis de adenocarcinoma endocervical. La paciente tuvo una buena evolución postoperatoria con remisión de los síntomas neurológicos.
\end{abstract}

Palabras clave: Cáncer de cuello uterino; Cérvix; Metástasis cerebral; Reporte de caso. (fuente: DeCS BIREME)

\begin{abstract}
Cervical cancer is the most frequent type of cancer in Peruvian women and in the general population of Peru. Brain metastases from cervical cancer are extremely rare with an incidence of 0.4 to $2.3 \%$ and only about 100 case reports in the medical literature. We report a case of a 52-year-old woman with a history of cervical cancer who, after 14 months of diagnosis, presented with headache, vomiting and loss of consciousness. In the brain MRI study, a contrast-enhancing lesion was evidenced in the right parieto-occipital region. A right parietal minicraneotomy was performed with stereotactic assistance and complete resection of the tumor. According to the report of the pathology service metastasis of endocervical adenocarcinoma is indicated. The patient had a good postoperative evolution with remission of neurological symptoms.
\end{abstract}

Key words: Cervical cancer; Cervix; Brain metastasis; Case report. (source: MeSH NLM)

\section{INTRODUCCIÓN}

El cáncer de cuello uterino es el segundo tipo de cáncer más frecuente en el mundo en la mujer luego del cáncer de mama. En las mujeres peruanas y en la población en general del Perú el cáncer de cuello uterino es el cáncer más frecuente y además implica el segundo lugar en mortalidad relacionada al cáncer en la mujer peruana'.

Las metástasis de cáncer de cuello uterino se producen generalmente en estadios avanzados de la enfermedad siendo su forma de diseminación más frecuente la local, linfática y con menos frecuencia la vía hematógena hacia órganos distantes siendo los más frecuentemente afectados el pulmón, ganglios supraclaviculares, hígado y huesos ${ }^{17,18}$.

Las metástasis cerebrales son extremadamente raras con una incidencia de 0.4 a $2.23 \%$ y sólo aproximadamente 100 reportes de casos en la literatura médica ${ }^{3,4,10,13,15,18}$.

'Residente de Neurocirugía. Servicio de Neurocirugía. Hospital Nacional Alberto Sabogal Sologuren - EsSalud, Lima-Perú.
${ }^{2}$ Médico Neurocirujano. Servicio de Neurocirugía. Hospital Nacional Alberto Sabogal Sologuren - EsSalud,Lima-Perú.

Citar como: Álvaro Alzamora-Jiménez, Alejandro Castillo-Gutierrez, Juan Coasaca-Torres. Metastasis cerebral de adenocarcinoma de cervix. [Caso Clínico].2018;18(3):120-126. (Octubre 2018). DOI 10.25176/RFMH.v18.n4.1740 
En el presente trabajo describimos el caso de una mujer de 52 años de edad con una metástasis cerebral de cáncer de cérvix en la región parieto-occipital derecha la cual fue resecada quirúrgicamente en su totalidad mediante craneotomía mínima y con asistencia de estereotaxia.

\section{PRESENTACIÓN DEL CASO}

En Octubre del 2017, una paciente de 52 años de edad con antecedente de adenocarcinoma de cuello uterino estadio IB que fue diagnosticada 14 meses antes y fue tratada mediante histerectomía radical y tratamiento adyuvante con quimioterapia, radioterapia y braquiterapia en otra institución, acude al servicio de emergencia del Hospital Alberto Sabogal Sologuren con un tiempo de enfermedad de 2 meses caracterizado por cefalea tipo opresiva de predominio bifrontal y vómitos recurrentes que aumentaron en intensidad y frecuencia. Al examen se encuentra una paciente despierta con Escala de Glasgow de 15, con hemiparesia izquierda (4/5). Se hospitaliza en el área de emergencia a cargo del Servicio de Neurocirugía para observación y completar estudio de imágenes. Se realiza una tomografía cerebral con contraste donde se evidencia una lesión pequeña captadora de contraste en la región parieto-occipital derecha (Fig. 1). Se amplía el estudio con una resonancia magnética cerebral (Fig. 2). Se evidencia una lesión parieto-occipital derecha heterogénea captadora de contraste en forma de anillo irregular con edema circundante extenso que produce efecto de masa y desviación de línea media hacia la izquierda. Se solicita una tomografía de tórax abdomen y pelvis como parte del estudio siendo el resultado negativo. Considerando el antecedente ginecológico se plantea el diagnóstico de metástasis cerebral solitaria parieto-occipital derecha por lo cual es programada para cirugía de resección total.

Se realiza una craneotomía parietal derecha mínima y mediante abordaje transulcal se logra la resección total de la lesión con asistencia estereotáxica para la identificación de los límites tumorales. Se evidencia una tumoración redondeada de $4 \times 4 \times 3 \mathrm{~cm}$ de color amarillento y consistencia sólida (Fig. 3 y Fig. 4).

El resultado del informe del Servicio de Patología indica el diagnóstico de Adenocarcinoma pobremente diferenciado de tipo endocervical con lo que se confirma el diagnóstico de metástasis cerebral de cáncer de cérvix. La paciente tolera la cirugía de forma adecuada y es dada de alta con buena evolución luego de 8 días para control por consulta externa de neurocirugía y continuar tratamiento adyuvante por el servicio de Oncología.

\section{DISCUSIÓN}

Las metástasis cerebrales de cuello uterino fueron descritas por primera vez por Henriksen en $1949^{19}$, desde esa fecha hasta la actualidad se han publicado pocos reportes de caso y además la literatura médica relacionada a la descripción de las características clínico-patológicas, del mejor tratamiento y el pronóstico de esta enfermedad es aún escasa.

La incidencia de metástasis cerebrales de diversos cánceres varía de $13.5 \%$ a 37\%, siendo los pulmones, mama, y melanoma las causas más frecuentes ${ }^{20,22}$. Los cánceres ginecológicos tienen baja tendencia de hacer metástasis al cerebro, de ellas la entidad más frecuente es el coriocarcinoma que ocupa un 35\% de las metástasis de este tipo ${ }^{21}$.

La incidencia de las metástasis cerebrales de cuello uterino es extremadamente rara con incidencias descritas en distintos trabajos que varían desde 0.4 a $2.23 \% \%^{3,4,10,13,15,18}$. Por otro lado Fetcko et al $^{3}$ describe que hasta el 3 al 10\% de pacientes con cáncer de cérvix tienen metástasis cerebral, además en su revisión de casos hasta el 2017 encontró sólo 31 reportes con un total de 39 pacientes, además 5 series de casos analizando un total de 50 pacientes y 4 revisiones retrospectivas involucrando 60 pacientes. Según nuestro conocimiento el presente trabajo es el primer reporte de caso relacionado al diagnóstico de metástasis cerebral secundaria a carcinoma de cuello uterino en el Perú.

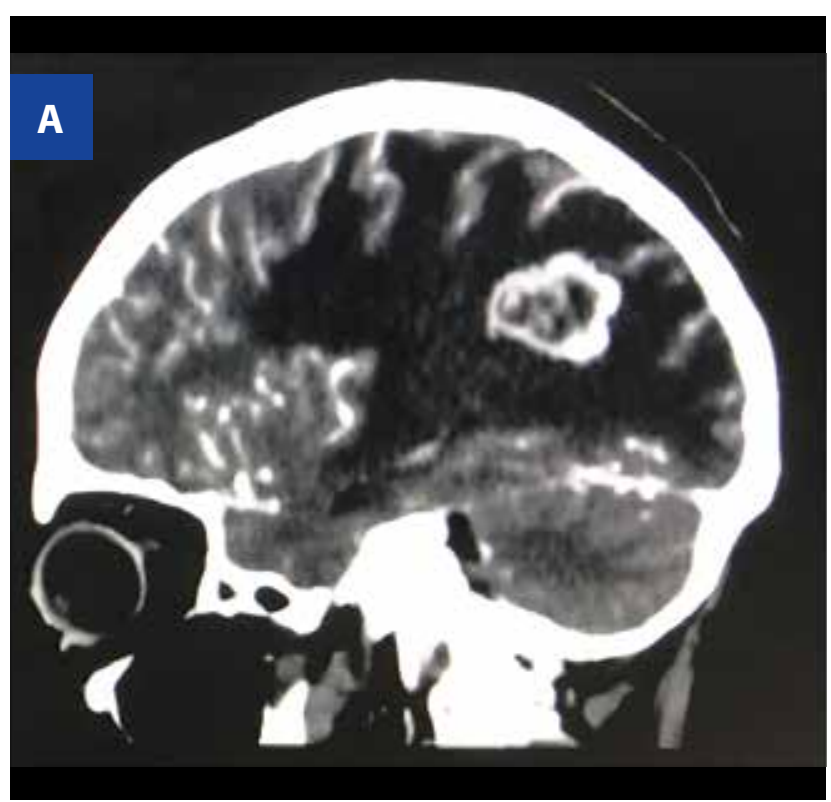




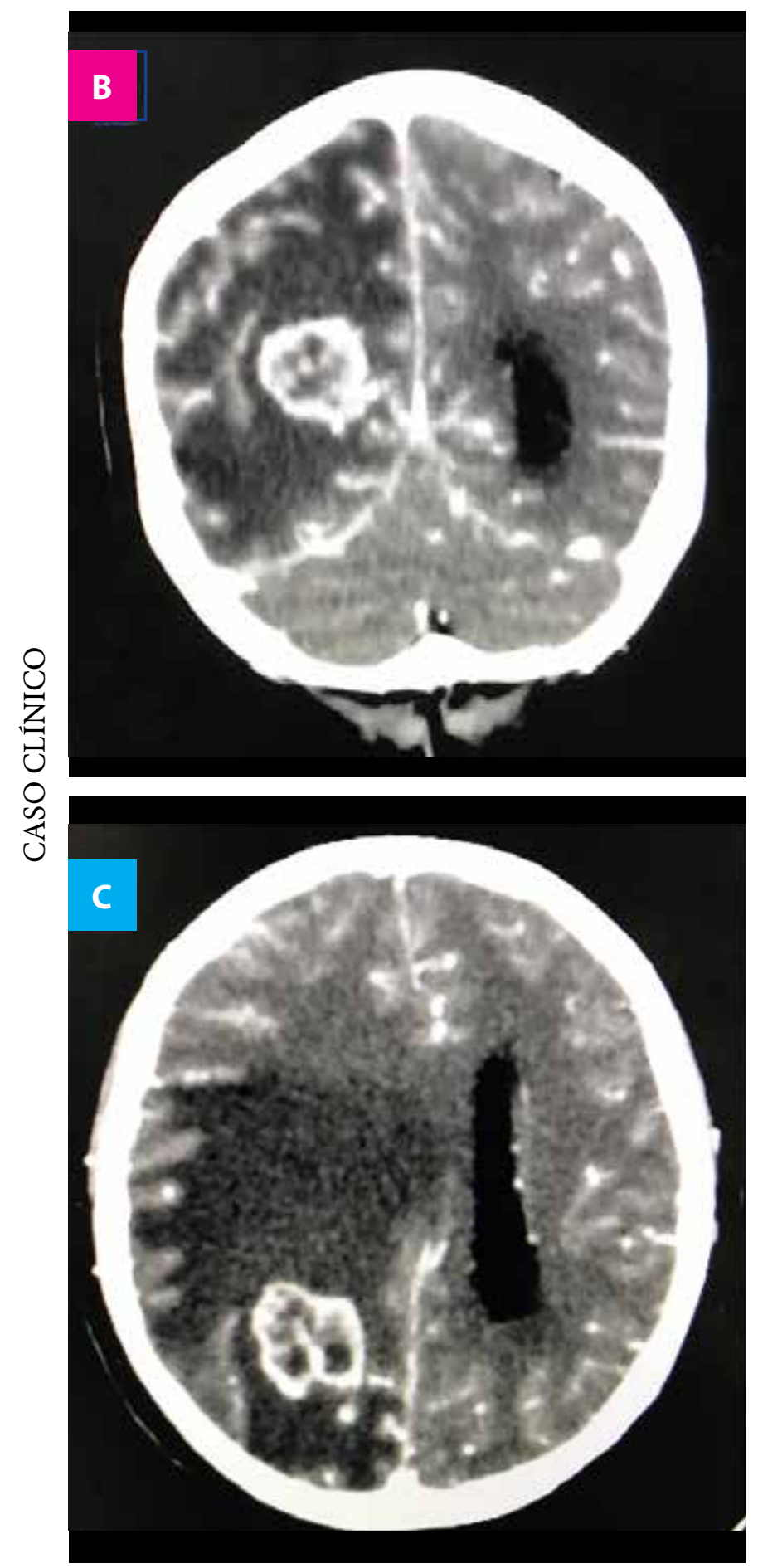

Figura 1. Tomografía cerebral con contraste en corte a) sagital b) coronal y c) axial. Se evidencia la tumoración parieto-occipital derecha con captación de contraste en forma de anillo irregular con centro hipodenso.

Según la literatura se describe que la ruta de diseminación de metástasis cerebral de cuello uterino se produce por vía hematógena, a través del sistema venoso vertebral ${ }^{15,18}$. Branch et al $^{25}$ postuló que el mecanismo de diseminación de las metástasis cerebrales se producen luego de su siembra hacia los pulmones esto coincide con la revisión de Fetcko et $\mathrm{al}^{3}$ que menciona que los pulmones son el lugar más frecuente de metástasis de cérvix. Sin embargo, se conoce que la presencia de células tumorales en la circulación cerebral no necesariamente conlleva a una metástasis, este hecho depende del sistema inmune del paciente, el número de émbolos tumorales, la neovascularización tisular y las características del tumor 6,14
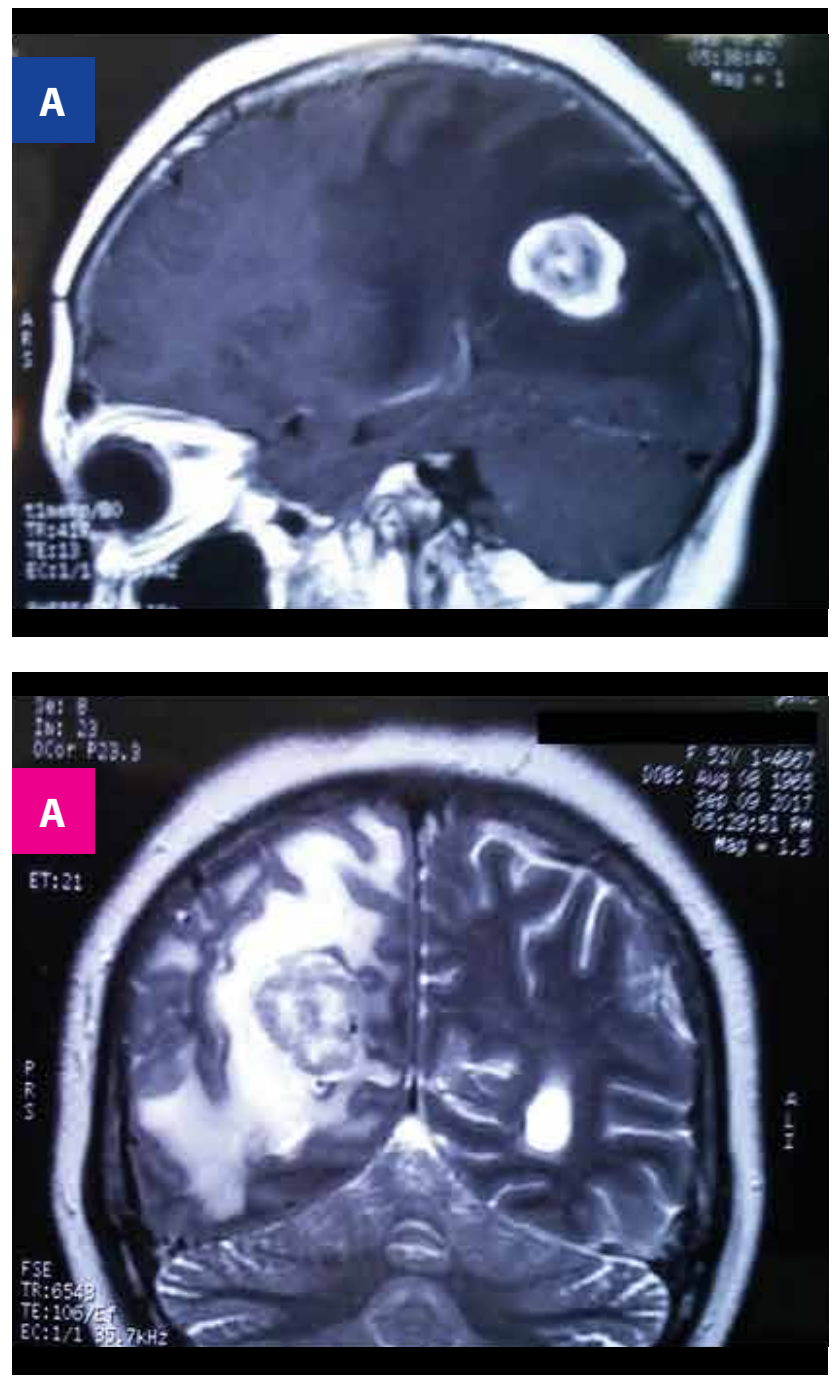

Figura 2. Resonancia magnética cerebral en secuencia a) $\mathrm{T} 1$ con contraste en corte sagital y b) T2 en corte coronal. Se confirma la lesión parieto-occipital derecha, captadora de contraste con edema cerebral extenso. 

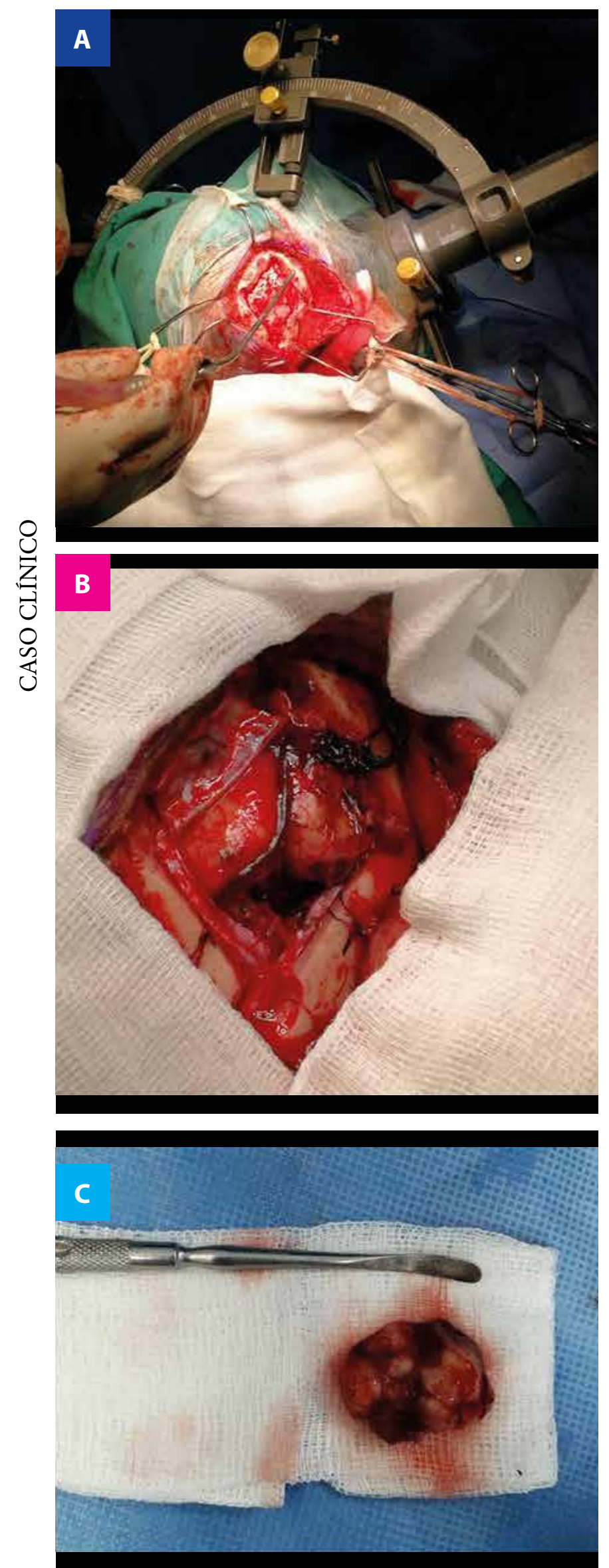

Figura 3. Fotografías intraoperatorias donde se evidencia a) el tamaño de la craneotomía y el marco de estereotaxia posicionado en la cabeza, b) abordaje transulcal y c) la pieza operatoria luego de ser resecada en su totalidad.
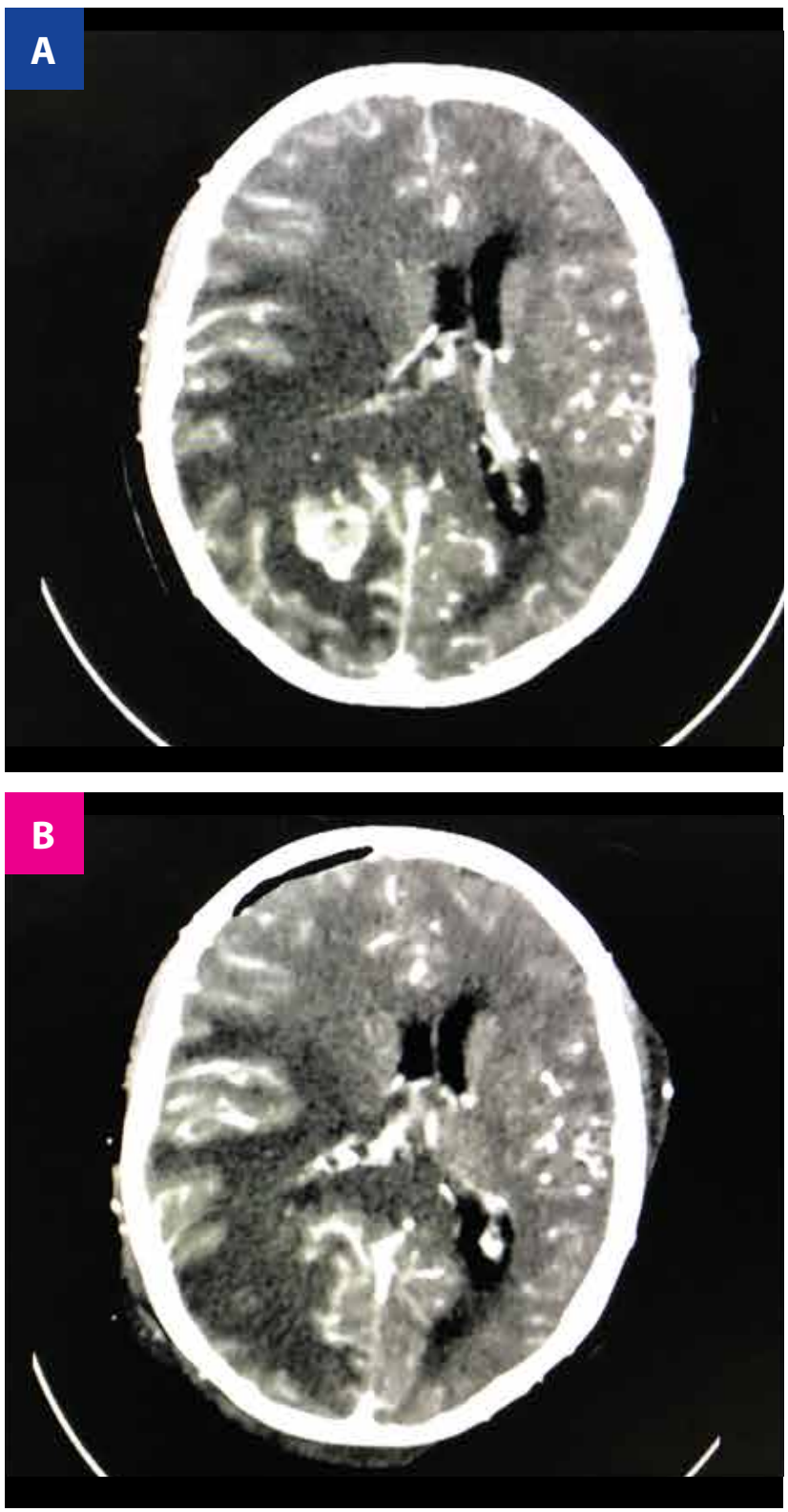

Figura 4. Tomografía cerebral con contraste en corte axial a) preoperatoria y b) en el postoperatorio inmediato donde se evidencia la resección completa de la lesión.

En cuanto al estadio de la enfermedad Chura et al $^{15}$ mostró que sólo el $25 \%$ de su serie de casos tuvo enfermedad avanzada (III y IV). Por su parte Nasu et $\mathrm{al}^{4}$ en su revisión sobre casos de metástasis cerebrales de origen ginecológico describe que la mayoría de pacientes con cáncer de ovario (85.7\%) y cuerpo de útero (69.3\%) tuvieron enfermedad avanzada (III,IV), pero que sólo el $35.7 \%$ de pacientes con cáncer de cuello uterino estuvo en edad avanzada. Esto concuerda con la revisión de Fetcko et al ${ }^{3}$ que encontró que $60 \%$ de los casos de la literatura hasta el año 2017 estuvieron en estadio I o II. Sin embargo, MahmoudAhmed et $\mathrm{al}^{5}$ postula que las metástasis cerebrales de cuello uterino tienen un comportamiento errático y que no puede ser predichas ni por el estadio de la enfermedad ni por la histología de la misma. Nuestro 
caso concuerda con la literatura pues se presentó una metástasis cerebral en una paciente con estadio IB de cáncer de cuello uterino.

En cuanto a la histología los reportes mencionan que el tipo de cáncer de cuello uterino más frecuente es pobremente diferenciado y de tipo escamoso ${ }^{3,7,14}$. Chura et $\mathrm{al}^{15}$ en su reporte describe 8 casos de tipo escamoso, 3 de adenocarcinomas y 1 de adenoescamoso. Se ha reportado casos de carcinomas de cérvix de tipo neuroendocrino que estarían aumentando en incidencia en cuanto a metástasis a cerebro ${ }^{6,7}$. Nuestro caso presenta una metástasis cerebral de adenocarcinoma de cuello uterino lo cual según la literatura es muy poco frecuente. Fetcko et $\mathrm{al}^{3}$ en los 31 reportes de casos y las 5 series de casos (total de 89 pacientes) sólo encontró el diagnóstico de metástasis cerebral por adenocarcinoma de cuello uterino en 22 casos. En nuestra revisión sólo logramos encontrar 3 casos con el diagnostico de metástasis cerebral por adenocarcinoma de cuello uterino ${ }^{16,17}$.

La edad de presentación metástasis cerebral es significativamente menor en pacientes con metástasis cerebral de cáncer de cuello uterino (50.3 años) comparado a otros cánceres de origen ginecológico (ovario 55.8 años y cuerpo de útero 60.9 años) ${ }^{7}$. Por otro lado Chura et al describe una edad media de presentación de 43.5 años (rango de 29-57 años) y Fetcko et al $^{3}$ menciona una edad media de 48 años con un rango de 29 a 87 años. Nuestro caso con 52 años de edad supone una edad acorde con la media descrita en la literatura médica. A su vez en nuestra revisión de casos encontramos una edad media al diagnóstico de 50 años $^{6-18}$.

El intervalo entre el diagnóstico de metástasis cerebral y el de cáncer de cuello uterino es variable. Nuestro caso presentó clínica neurológica y se hizo el diagnóstico de metástasis cerebral luego de 14 meses del diagnóstico de la neoplasia ginecológica lo que se aproxima a lo encontrado por otros autores. Mahmoud-Ahmed et al $^{5}$ reporta una media de 12 meses. Chura et al ${ }^{15}$ menciona 17.5 meses de media con rango de 1.1 a 96.1 meses. Sin embargo Robinson et al describió hasta 8 años con media de 30 meses. Nasu et al en su revisión muestra que el intervalo entre el diagnóstico inicial de cáncer ginecológico y el de metástasis cerebral es mayor en el caso del cuello uterino (36.3 meses) comparado a los casos de ovario (25.2 meses) y cuerpo de útero (25.1 meses) ${ }^{4}$ Brown et $\mathrm{al}^{23}$ reportó un caso excepcional en el cual se diagnosticó una metástasis cerebral occipital por presentar síntomas neurológicos a una paciente con diagnóstico de cáncer de cuello uterino 2 semanas antes. Otro caso singular es el reportado por Sato et $\mathrm{al}^{7}$ quien describe una paciente diagnosticada inicialmente de una lesión cerebral frontal derecha por presentar cefalea, vértigo y amnesia que luego de completar los estudios de tomografía computada con contraste de todo el cuerpo como parte del estudio de una posible metástasis se evidencia una lesión pélvica que finalmente fue diagnosticada como carcinoma de cérvix siendo la neoplasia primaria y la lesión cerebral una metástasis de la misma.

Las manifestaciones clínicas más frecuentes de metástasis cerebrales de cuello uterino son según la literatura, cefalea, hemiparesia, paresia facial, trastorno de conciencia y convulsiones ${ }^{18}$ En nuestro caso la paciente presentó cefalea, vómitos y pérdida de conciencia. Sin embargo, como es bien conocido, la sintomatología en las patologías neurológicas depende de la ubicación de la lesión. La localización más frecuente de metástasis cerebrales es en la región supratentorial, específicamente en los hemisferios cerebrales $4,5,10,13,14$ y con más frecuencia en el lóbulo frontal ${ }^{16}$. Fetcko et $\mathrm{al}^{3}$ describe que si bien la localización infratentorial es menos frecuente el área más común dentro de ella es el cerebelo. En nuestra revisión de casos reportados encontramos que la mayoría son supratentoriales, encontrando sólo 2 casos de metástasis infratentorial y 2 casos de lesión infra y supratentorial. Además, encontramos 1 caso de metástasis intramedulares múltiples y 1 caso de metástasis parénquima cerebral supratentorial con extensión a cráneo y cuero cabelludo adyacente ${ }^{6-18}$. Mahmoud-Ahmed et $\mathrm{al}^{5}$ en su revisión de 6 casos encuentra que 4 de ellos presentaron metástasis múltiples distribuidos en los hemisferios cerebrales ( 2 casos) o en hemisferios cerebrales y cerebelosos ( 2 casos). Sólo 2 casos tuvieron metástasis solitarias confinadas a un hemisferio cerebral. Nasu et al reportó que sólo el $33.3 \%$ de metástasis cerebrales de cuello uterino son solitarias, siendo en su mayoría múltiples. Fetcko et al $^{3}$ encontró en su revisión que el $55 \%$ de pacientes tuvieron lesiones múltiples y $45 \%$ solitarias. En la revisión de Chura et al ${ }^{15}$ de 12 pacientes, 4 tuvieron lesiones cerebrales solitarias, 6 tuvieron lesiones cerebrales múltiples y 2 tuvieron diseminación leptomeníngea. En nuestra revisión de casos clínicos encontramos 7 reportes de lesiones solitarias y 5 de lesiones múltiples ${ }^{6-18}$.

Sato et $\mathrm{al}^{7}$ describen que aproximadamente en la mitad de los casos reportados en la literatura las metástasis cerebrales de cuello uterino son la única lesión secundaria. Sin embargo, Fetcko et al $^{3}$ incluye 
en su revisión que la mayoría de casos descritos presentan otra lesión metastásica al ser diagnosticados de metástasis cerebral de cuello uterino y que sólo una pequeña fracción de los casos tiene lesiones metastásicas cerebral como única lesión secundaria. En nuestra revisión de casos reportados 4 casos presentaron otra metástasis aparte de la lesión cerebral siendo éstas en epiplón, intramedular, pulmón, cráneo y cuero cabelludo 8,12,13,17. En el caso presentado por nosotros la paciente presentó una lesión solitaria, supratentorial en la región parieto-occipital derecha y además ésta fue la única metástasis identificada al momento del diagnóstico.

En cuanto al pronóstico y tratamiento de las metástasis cerebrales de cuello uterino, MahmoudAhmed et $\mathrm{al}^{5}$, reportó en su revisión de 6 casos que 1 caso fue tratado sólo con radiocirugía estereotáxica, 3 fueron operados y posteriormente recibieron terapia de radiación holocraneal, 1 sólo recibió terapia de radiación holocraneal y 1 recibió terapia de radiación holocraneal además de radiocirugía estereotáxica. Los pacientes tratados con cirugía tuvieron una sobrevida media de 8.25 meses, mientras que los pacientes tratados con terapia de radiación holocraneal con o sin radiocirugía estereotáxica tuvieron una sobrevida media de 3.75 meses. Por su parte Chura et $\mathrm{al}^{15}$, reportó en su revisión de 12 casos que 8 pacientes recibieron terapia de radiación holocraneal y esteroides, 3 recibieron sólo esteroides y 1 fue sometido a cirugía seguida de irradiación. Todos los pacientes que recibieron terapia de radiación holocraneal experimentaron mejoría de sus síntomas. La sobrevida media de los pacientes fue de 2.3 meses. 5 pacientes que recibieron quimioterapia luego de radioterapia tuvieron una sobrevida de 4.4 meses comparado a 0.9 meses en aquellos que no recibieron tratamiento adicional luego de la radioterapia. Con esto el autor concluye que a pesar del pobre pronóstico en este tipo de pacientes la quimioterapia mejoraría su sobrevida. En su extensa revisión de Nasu et $\mathrm{al}^{4}$ describe que la sobrevida media en el grupo de pacientes con metástasis cerebral por cáncer de cuello uterino (5.0 meses) es menor que la de otras entidades ginecológicas como la de ovario (12.5 meses) y cuerpo del útero (6.2 meses). La tasa de sobrevida a los 2 años es de $4.8 \%$ para los casos de cáncer e ovario en comparación a los $19.7 \%$ y $6.1 \%$ de los casos de cáncer de ovario y cuerpo de útero respectivamente. Nasu además describe como factores favorables independientes de sobrevida, el puntaje en la escala de Karnofsky (KPS) >70, metástasis cerebral solitaria, ausencia de enfermedad extracraneal, cirugía craneal, radioterapia craneal y quimioterapia.
Actualmente no existe un tratamiento estandarizado para las metástasis cerebrales de cuello uterino, y diversos tratamientos y combinaciones de opciones terapéuticas se han utilizado como la resección quirúrgica, la terapia de irradiación holocraneal, quimioterapia y radiocirugía estereotáctica. Sin embargo, el tratamiento debe ser individualizado y multimodal. Se sugiere radiocirugía estereotáctica o terapia de radiación holocraneal para metástasis solitarias seguido de quimioterapia en enfermedad sistémica. La resección quirúrgica con terapia de radiación holocraneal es sugerida para lesiones solitarias sin enfermedad sistémica y la terapia de radiación holocraneal o la radiocirugía estereotáctica junto a esteroides seguido de quimioterapia para manejo paliativo ${ }^{5,15,16}$. En la revisión realizada por Cordeiro et al $^{14}$ encontró que el tratamiento más común según la literatura fue la radiación holocraneal (17\%) y la cirugía seguida de radiación holocraneal (13\%). La radioterapia adyuvante postoperatoria ha llevado a mejora de la sobrevida, mejoría del estado neurológico y menor recurrencia en lesiones del sistema nervioso central que la radioterapia como único tratamiento. La quimioterapia juega un rol importante en el tratamiento del cáncer de cérvix, específicamente el cisplatino, sin embargo, su efecto en el resultado clínico de las metástasis cerebrales de cáncer de cérvix aún no claro, pero podría ser utilizado en los casos de lesiones múltiples.

En el caso de nuestra paciente con un KPS de $60 \%$, en la cual se consideró como mejor tratamiento la resección quirúrgica total de una lesión solitaria y sin enfermedad extracraneal. En sus controles actuales (luego de 2 meses de operada) la encontramos con KPS de 100 y siendo evaluada por el servicio de Oncología para iniciar el tratamiento adyuvante de quimioterapia y radioterapia. Mantendremos un seguimiento estricto de la paciente y confiamos que sus factores pronósticos junto con el pronto inicio del tratamiento adyuvante influenciarán positivamente en su sobrevida.

La literatura médica sobre las metástasis de cuello uterino aún es escasa y depende de su investigación el mejor entendimiento y por tanto la mejora en el tratamiento a fin de ofrecer una mejor calidad de vida y mayor sobrevida en casos determinados. Nuestro caso muestra una presentación poco frecuente de metástasis de cáncer de cuello uterino que fue tratada quirúrgicamente de forma exitosa con remisión de síntomas neurológicos y evolución favorable en la actualidad. 
Recomendamos la correcta evaluación neurológica y según ello la exploración con imágenes de ayuda al diagnóstico en la región cerebral en pacientes con cáncer de cuello uterino con la finalidad del diagnóstico y tratamiento oportuno de posibles metástasis cerebrales.

\section{CONCLUSIÓN}

La metástasis cerebral de cuello uterino es una patología poco frecuente y con pronóstico poco favorable que debe ser considerada en los pacientes con este antecedente neoplásico que presenten manifestaciones clínicas de tipo neurológico. El presente trabajo demuestra que en casos determinados los pacientes pueden beneficiarse de tratamiento quirúrgico con buenos resultados postoperatorios ofreciendo una mejoría de la calidad de vida. Sin embargo, es recomendable el tratamiento multimodal para favorecer la mayor sobrevida.
Contribuciones de autoría: Los autores participaron en la generación, redacción y aprobación final del artículo original.

\section{Financiamiento: Autofinanciado.}

Conflicto de interés: Los autores declaran no tener conflicto de interés en la publicación de este articulo.

Recibido: 21 de junio del 2018

Aprobado: 25 de setiembre del 2018

\section{Correspondencia: Álvaro Alzamora-Jiménez}

Dirección: INICIB, Facultad de Medicina Humana, Edificio I-208. 2do piso. Avenida Benavides 5440, Surco, Lima-Perú.

Teléfono: 708-0000 / Anexo: 6016

Correo:alzamorajimenez@gmail.com

\section{REFERENCIAS BIBLIOGRÁFICAS}

1. Ferlay J, Soerjomataram I, Ervik M, Dikshit R, Eser S, Mathers C, et al. GLOBOCAN 2012 v1.0, Cancer Incidence and Mortality Worldwide: IARC CancerBase No. 11 Lyon, France: International Agency for Research on Cancer; 2013. Disponible en: $\underline{\text { http://globocan.iarc.fr }}$

2. Piñeros M. et al. Cancer patterns, trends, and transitions in Peru: a regional perspective. Lancet Oncol 2017; 18: e573-86

3. Fetcko K, Gondim D, Bonnin J, Fey M. Cervical cancer metastasis to the brain: A case report and review of literature Surgical Neurology International 2017, 8:181

4. K. Nasu et al. Clinicopathologic features of brain metastases from gynecologic malignancies: A retrospective study of 139 cases (KCOG G1001s trial) Gynecologic Oncology 128 (2013) 198-203

5. Mahmoud-Ahmed AS, Suh JH, Barnett GH, Webster KD, Kennedy AW Tumor distribution and survival in six patients with brain metastases from cervical carcinoma. Gynecol Oncol 2001; 81: 196-200

6. Amita M, Sudeep G, Rekha W, Yogesh K, Hemant T. Brain metastasis from cervical carcinoma-a case report. Med Gen Med 2005;7:26.

7. Sato Y. et al.Uterine cervical cancer with brain metastasis as the initial site of presentation. J. Obstet. Gynaecol. Res. Vol. 41, No. 7: 1145-1148, July 2015

8. Vitorino-Araujo J.L.et al Scalp, skull and brain metastasis of squamous cell carcinomaof the cervix - a rare entity British Journal of Neurosurgery, August 2013; 27(4): 519-520

9. Chekrine T, Hassouni A, Jouhadi H, Sahraoui S, Bouchbika Z et al Métastase cérébrale d'un carcinome du col utérin. Pan African Medical Journal. 2013; 14: 114

10. Setoodeh R, Hakam A, Yuan Shan Y. Cerebral metastasis of cervical cancer, report of two cases and review of the literature. Int J Clin Exp Pathol 2012;5(7):710-714

11. S. H. Park et al.Brain metastasis from uterine cervical cáncer. J. Obstet. Gynaecol. Res. Vol. 36, No. 3: 701-704, June 2010

12. Peters $P$, Bandi H, Efendy J, Perez-Smith A, Olson S. Rapid growth of cervical cancer metastasis in the brain. Case Reports / Journal of Clinical Neuroscience 17 (2010) 1211-1212
13. Ding D.C, Chu T.Y. Brain and Intramedullary Spinal Metastasis from Squamous Cell Cervical Carcnioma. Taiwan J Obstet Gynecol • December $2010 \cdot$ Vol $49 \cdot$ No 4

14. Agrawal A, Kumar A, Sinha A K, Kumar M, Pandey S R, Khaniya S. Intracranial metastases from carcinoma of the cervix. Singapore Med J 2007; 48(5) : e154

15. Chura JC, Shukla K, Argenta PA. Brain metastasis from cervical carcinoma. Int J Gynecol Cancer 2007;17:141 6.

16. Graciolli Cordeiro J, Monte-Serrat Prevedello D, Da Silva DItzel L, Pereira C, Araujo J. Cebrebral Metastasis of Cervical Uterine Cancer. Arq Neuropsiquiatr 2006;64(2-A):300-302

17. Nagar Y.S, Shah N, Rawat S, Kataria T. Intracranial metastases from adenocarcinoma of cervix: a case reportInt J Gynecol Cancer 2005, 15, $561-563$

18. Robinson J, Morris M. Cervical Carcinoma Metastatic To The Brain Gynecologic Oncology 66, 324-326 (1997).

19. Henriksen E: The lymphatic spread of carcinoma of the cervix and of the body of the uterus. Am J Obstet Gynecol 58:924-942, 1949

20. Stortbecker TP. Metastatic tumors of the brain from a neurosurgical point of view: a follow-up of 158 cases. J Neurosurg. 1954;11:84-111.

21. Yordan LL, Jr, Schlaerth J, Gaddis O, Morrow CP. Radiation therapy in the management of gestational choriocarcinoma metastatic to the central nervous system. Obstet Gynecol. 1987;69:627-630.

22. Al-Shamy G, Sawaya R. Management of brain metastases: the indispensable role of surgery. J Neurooncol 2009;92:275-82.

23. Brown lii JV, Epstein HD, Kim R, Micha JP, Rettenmaier MA, Mattison JA, et al. Rapid manifestation of CNS metastatic disease in a cervical carcinoma patient: A case report. Oncology 2007;73:273 6.

24. Ogawa K, Yoshii Y, Aoki Y, Nagai Y, Tsuchida Y, Toita T, et al. Treatment and prognosis of brain metastases from gynecological cancers. Neurol Med Chir 2008;48:57-63.

25. Branch BC, Henry J, Vecil GG. Brain metastases from cervical cancer — A short review. Tumori 2014;100:e171 9. 